\title{
SUBALGEBRAS OF GROUP ALGEBRAS
}

\section{ROGER RIGELHOF}

I. Let $G$ be a locally compact group and $m$ its Haar measure. For any $m$-measurable subset $S$ of $G$, let $L(S)$ be the subspace of $L^{1}(G)$ consisting of elements $f$ such that $\int_{G \backslash S}|f| d m=0$. If $S$ is a subsemigroup then $L(S)$ is a subalgebra of $L^{1}(G)$. Various papers ([4], [5] and [7]) have been devoted to the study of $L(S)$ and to the question of whether there is a subsemigroup $T$ such that $L(S)=L(T)$ whenever $L(S)$ is an algebra. In [5] it is shown that this is the case whenever $S$ is contained in a $\sigma$-compact subset. A related problem is the following. Let $d S$ be the set of all $x$ in $G$ such that each measurable neighborhood of $x$ meets $S$ in a set of positive measure. Whenever $L(S)$ is an algebra, $d S$ is a subsemigroup [7], but it need not be true that $L(S)=L(d S)$. In this paper we show that in certain cases $L(S)=L(d S)$. Using this we give very easy proofs of some of the results in [4] and [7].

Let $M(G)$ be the Banach *-algebra of bounded regular Borel measures on $G$. (We follow [3] in the definition of Borel subsets etc.) For a Borel subset $S$ of $G$, let $M(S)$ be the set of $\mu \in M(G)$ with $|\mu|(G \backslash S)=0$. Suppose that $S$ is a measurable subsemigroup of $G$ so that $L(S)$ is a subalgebra of $L^{1}(G)$. Let $L(S)^{\pi}$ be the algebra of left multipliers of $L(S)$, i.e. the algebra of bounded linear maps $\pi$ of $L(S)$ into itself such that $\pi(f * g)=\pi f * g$. Let $S^{\pi}=\{x \in G: x S \backslash S$ is locally null $\}$. In this paper we show that if $G$ is abelian then $S^{\pi}$ is closed and that $M\left(S^{\pi}\right) \subset L(S)^{\pi}$, and under certain additional hypotheses $M\left(S^{\pi}\right)$ $=L(S)^{x}$. This question has been considered by Birtel in [1] and T. A. Davis in [2]. The question is of some interest since Davis (op. cit.) showed that for abelian $G$, the Wiener-Pitt phenomenon occurs for measures in $L(S)^{\pi}$.

II. Let $C_{0}(G)$ be the Banach space of continuous complex-valued functions on $G$ which "vanish at infinity." Let $f$ be a function on $G$; the support of $f, \operatorname{Supp}(f)$ is the closure of $\{x \in G: f(x) \neq 0\}$. Let $K(G)$ be the subspace of $C_{0}(G)$ of functions whose support is compact. Let $\mu$ be a measure on $G$. The support of $\mu, \operatorname{Supp}(\mu)$ is the smallest closed subset of $G$ whose complement has $|\mu|$-measure zero.

It is well known that $L^{1}(G)$ may be identified with the subspace of $M(G)$ of measures that are absolutely continuous with respect to the Haar measure $m$, and as such is a two-sided closed ideal in $M(G)$. The $s o$-topology (resp. $s o_{r}$-topology) on $M(G)$ is the coarsest topology

Received by the editors February 13, 1969. 
such that for every $\lambda$ in $L^{1}(G)$ the mapping $\mu \mapsto \mu * \lambda$ (resp. $\mu \mapsto \lambda * \mu$ ) is a continuous mapping of $M(G)$ into $L^{1}(G)$.

The first proposition is a well-known property of the support of a measure, and we omit the proof.

Proposition 1. Let $S$ be a closed subset of $G$ and let $\mu \in M(G)$. Then $\mu$ is in $M(S)$ if and only if $\mu(f)=0$ for each $f \epsilon K(G)$ with $S \cap \operatorname{Supp}(f)=\varnothing$.

For $x \in G$, let $\epsilon_{x}$ be the Dirac measure at $x$, i.e. $\epsilon_{x}(f)=f(x)$ for $f \in C_{0}(G)$.

Proposition 2. Let $S$ be a Borel subset of $G . S$ is closed if and only if $M(S)$ is so-closed.

Proof. Suppose that $M(S)$ is so-closed and that $a$ is in the closure of $S$. The mapping $x \mapsto \epsilon_{x}$ is so-continuous $[3,20.4]$ so that $\epsilon_{a} \in M(S)$. This means that $\epsilon_{a}(G \backslash S)=0$ and consequently $a \in S$. Thus $S$ is closed.

Now suppose that $S$ is closed and let $\mu \in \mathrm{Cl}_{s o} M(S)$. There is a net $\left(\mu_{j}: j \in J\right)$ in $M(S)$ such that $\mu_{j} \stackrel{s o}{\rightarrow} \mu$, and $\operatorname{Supp}\left(\mu_{j}\right) \subset S$. Let $f$ in $K(G)$ be such that $S \cap \operatorname{Supp}(f)=\varnothing$. There is a symmetric neighborhood $V$ of $e$ in $G$ such that $S \cap \operatorname{Supp}(f) V=\varnothing[3,4.10]$. Let $\epsilon>0$ be given, then by the uniform continuity of $f$ there is a neighborhood $U$ of $e$ in $G$ such that $|f(x y)-f(x)| \leqq \epsilon / 2\|\mu\|$ for all $x$ in $G$ and $y$ in $U$. Let $\lambda$ be a positive measure in $L^{1}(G)$ such that $\operatorname{Supp}(\lambda) \subset U \cap V$, and $\|\lambda\|=1$. Then $\left|\int f(x y) d \lambda(y)-f(x)\right| \leqq \epsilon / 2|| \mu \|$ so that $|\mu * \lambda(f)-\mu(f)|$ $\leqq \epsilon / 2$. If $y \in V$ and $x \in G$ is such that $f(x y) \neq 0$ then $x \in \operatorname{Supp}(f) V^{-1}$ $C \operatorname{Supp}(f) V$, consequently $\int_{V} f(x y) d \lambda(y)=0$ whenever $x \in S$. Thus $\mu_{j} * \lambda(f)=0$. Since $\lambda \in L^{1}(G)$ there is a $j_{0}$ such that $j \geqq j_{0} \Rightarrow\left\|\mu_{j} * \lambda-\mu * \lambda\right\|$ $\leqq \epsilon / 2\|f\|$. Thus for $j \geqq j_{0}$, we have

$$
\begin{aligned}
|\mu(f)| & \leqq|\mu(f)-\mu * \lambda(f)|+\left|\mu_{j} * \lambda(f)-\mu * \lambda(f)\right|+\left|\mu_{j} * \lambda(f)\right| \\
& \leqq \epsilon / 2+\left\|\mu_{j} * \lambda-\mu * \lambda\right\|\|f\| \\
& \leqq \epsilon .
\end{aligned}
$$

Therefore we must have $\mu(f)=0$ for any $f \in K(G)$ with $S \cap \operatorname{Supp}(f)$ $=\varnothing$. By Proposition $1, \mu$ is in $M(S)$.

REMARK. Let $\sigma\left(M(G), C_{0}(G)\right)$ be the coarsest topology on $M(G)$ such that for each $f \in C_{0}(G)$, the map $\mu \mapsto \mu(f)$ is continuous. It is not difficult to show that a Borel subset $S$ of $G$ is closed if and only if $M(S)$ is $\sigma\left(M(G), C_{0}(G)\right)$-closed.

III. Let $S$ be an $m$-measurable subset of $G$, define $d S \subset G$ by $d S=\{x \in G$ : if $U$ is an open neighborhood of $x$, then $m(U \cap S)>0\}$. If $S$ is locally null then $L(S)=(0)$. To avoid this trivial case we shall 
suppose throughout that $S$ is not locally null and then $d S \neq \varnothing$. Note that $d S$ is always closed and that $L(S)=L(S \cap d S)$ [7].

Theorem 1. Let $S$ be an m-measurable subset of $G$. Then $L(S)$ and $L(d S)$ are dense in $M(d S)_{s \text { e. }}$.

Proof. Since $d S$ is closed, $M(d S)$ is so-closed (Proposition 2) and since $L(S) \subset L(d S)$ it suffices to show that $M(d S) \subset \mathrm{Cl}_{s o} L(S)$. Let $x \in d S, \lambda \in L^{1}(G)$ and $\epsilon>0$ be given. By $[3,20.15]$ there is a neighborhood $U$ of $e$ such that $\|\mu * \lambda-\lambda\| \leqq \epsilon$ for every positive measure $\mu \in M(G)$ with $\|\mu\|=1$ and $\mu(G \backslash U)=0$. Let $V=x U$, then $V$ is a neighborhood of $x$. Let $\mu \in L(S \cap V)$ be a positive measure with $\|\mu\|=1$. Then $\epsilon_{x^{-1}} * \mu(G \backslash U)=0$ and $\left\|\epsilon_{x^{-1}} * \mu\right\|=1$. Therefore

$$
\left\|\mu * \lambda-\epsilon_{x} * \lambda\right\|=\left\|\epsilon_{x-1} * \mu * \lambda-\lambda\right\| \leqq \epsilon .
$$

Clearly $\mu \in L(S)$ so that $\epsilon_{x} \in \mathrm{Cl}_{s o} L(S)$. Thus $x \in d S$ implies $\epsilon_{x} \in \mathrm{Cl}_{s o} L(S)$. By Proposition 2 of [6], each $\lambda \in M(d S)$ is an so-adherence point of the linear span of $\left\{\epsilon_{x}: x \in d S\right\}$ so it follows that $M(d S) \subset \mathrm{Cl}_{s \bullet} L(S)$.

Proposition 3. Let $S$ be an m-measurable subset of $G$. If $L(S)$ is an algebra then $L(d S) * L(d S) \subset L(S)$.

Proof. Let $\mu \in L(d S)$ and let $\left(\mu_{j}: j \in J\right) \subset L(S)$ be a net such that $\mu_{j} \stackrel{s o}{\rightarrow} \mu$. Let $\lambda \in L(S)$ then $\mu_{j} * \lambda \in L(S)$ and $\mu_{j} * \lambda$ converges in the norm to $\mu * \lambda$. Therefore $\mu * \lambda \in L(S)$ since $L(S)$ is norm closed. This shows that $L(d S) * L(S) \subset L(S)$. Theorem 1 remains true if we replace the $s o$-topology by the $s o_{r}$-topology. Consequently for $\lambda \in L(d S)$ there is a net $\left(\lambda_{j}: j \in J\right)$ in $L(S)$ such that $\lambda_{j} \stackrel{\text { sor }}{\rightarrow} \lambda$. For $\mu \in L(d S)$ we have $\mu * \lambda_{j} \in L(S)$ and $\mu * \lambda_{j}$ converges to $\mu * \lambda$ in the norm. This means that $\mu * \lambda \in L(S)$ which proves the proposition.

Corollary 1. If $L(S)$ is an algebra and if $e \in d S$, then $L(d S)=L(S)$.

Proof. This follows from the proposition, since $e \in d S$ implies that $L(d S)$ has an approximate unit.

In [7] Simon showed that if $L(S)$ is a nontrivial *-subalgebra then $d S$ is a subgroup of $G$. Using this and Corollary 1, we have

Corollary 2. Suppose that $L(S)$ is a *-subalgebra, then $L(S)=L(d S)$.

Corollary 3. Suppose that $G$ is compact. If $L(S)$ is a subalgebra of $L^{1}(G)$ then $d S$ is a closed subgroup of $G$ and $L(S)=L(d S)$.

ProOF. A closed subsemigroup of a compact group is necessarily a subgroup. 
Definition. Let $S$ be a measurable subset of $G$. If $L(S)$ is an algebra we shall follow [7] and call $L(S)$ a vanishing algebra. A vanishing algebra is called a maximal vanishing algebra if it is a proper subalgebra and if for every vanishing algebra $L(T), L(S) \subset L(T)$ implies $L(S)=L(T)$ or $L(T)=L^{1}(G)$.

We now give a very easy proof of a result due to Liu [4, Theorem 5].

THEOREM 2. If $L(S)$ is a maximal vanishing algebra, then $d S$ is a closed subsemigroup and $L(S)=L(d S)$.

Proof. We need only show $L(S)=L(d S)$. If $L(S) \neq L(d S)$ then by the maximality of $L(S), L(d S)=L^{1}(G)$, thus $d(d S)=d S$ is a subgroup and by Corollary $1, L(d S)=L(S)$ a contradiction.

IV. In this section we assume throughout that $G$ is a locally compact abelian group.

Proposition 4. Let $\pi \in L(S)^{\pi}$, then given $x \in d S$, there is a measure $\mu \in M(d S)$ such that for each $f \in L(S), \boldsymbol{\epsilon}_{x} * \pi f=\mu * f$.

Proof. Let $\left(e_{j}: j \in J\right)$ be a norm bounded net in $L(S)$ such that $e_{j} \stackrel{s 0}{\rightarrow} \epsilon_{x}$. Then $\left(\pi e_{j}: j \in J\right)$ is a norm bounded net in $M(G)$ and there is therefore a subnet $\left(\pi e_{j(i)}\right)$ and a $\mu \in M(G)$ such that $\pi e_{j(i)} \stackrel{s o}{\rightarrow} \mu$ (here $\sigma$ is the $\sigma\left(M(G), C_{0}(G)\right)$-topology). Since convolution is $\sigma\left(M(G), C_{0}(G)\right)$ continuous [6] we have for $f$ in $L(S), \pi e_{j(i)} * f_{\rightarrow}^{\sigma} \mu * f$. On the other hand convolution is also so-continuous, so that

$$
\pi e_{j(i)} * f=\pi\left(e_{j(i)} * f\right)=e_{j(i)} * \pi f \stackrel{s o}{\rightarrow} \epsilon_{x} * \pi f .
$$

Thus we have $\epsilon_{x} * \pi f=\mu * f$. To complete the proof we need to show that $\mu \in M(d S)$, and this follows since $M(d S)$ is $\sigma\left(M(G), C_{0}(G)\right)$ closed (remark following Proposition 2) and $L(S) \subset M(d S)$.

Proposition 5. Let $S$ be a subsemigroup of $G$ with nonzero Haar measure and let $S^{\pi}=\{x \in G: x S \backslash S$ is locally null $\}$. Then $S^{\pi}$ is closed.

Proof. By Proposition 4, $L(S)^{x}=\{\mu \in M(G): \mu * f \in L(S)$ for all $f \in L(S)\}$ so it follows that $L(S)^{\pi}$ is so-closed since $L(S)$ is norm closed. It is clear that $x \in S^{\pi}$ if and only if $\epsilon_{x} \in L(S)^{\pi}$. By Proposition 2 of [6] $x_{j} \rightarrow x$ if and only if $\epsilon_{x_{j}} \rightarrow \epsilon_{x}$ in the so-topology. It follows that $S^{\pi}$ is closed.

THEOREM 3. Let $S$ be a subsemigroup of $G$ having nonzero Haar measure. Then $M\left(S^{\pi}\right) \subset L(S)^{\pi}$ and if $e \in d S$ then $M\left(S^{\pi}\right)=M(d S)=L(S)^{\pi}$. 
Proof. Since $S^{\pi}$ is closed, $\mu \in M\left(S^{\pi}\right)$ if and only if $\operatorname{Supp}(\mu) \subset S^{\pi}$ so it follows from Proposition 2 of [6] that each $\mu$ in $M\left(S^{\pi}\right)$ is an so-adherence point of the linear span of $\left\{\epsilon_{x}: x \in S^{\pi}\right\}$. Each $\epsilon_{x}$ in $M\left(S^{\pi}\right)$ is in $L(S)^{\pi}$ so that $M\left(S^{\pi}\right) C L(S)^{\pi}$ since both $M\left(S^{\pi}\right)$ and $L(S)^{\pi}$ are so-closed (Propositions 2 and 5).

If $e \in d S$ then by Proposition $4, L(S)^{\star} \subset M(d S)$. It follows from Proposition 3 that $M(d S) \subset M\left(S^{\pi}\right)$.

Remark. T. A. Davis [2] states that $L(S)^{\pi} \subset M\left(S^{\pi}\right)$ (Theorem 3.5) however it appears that his proof is not complete.

\section{REFERENCES}

1. F. T. Birtel, On a commutative extension of a Banach algebra, Proc. Amer. Math. Soc. 13 (1962), 815-822.

2. T. A. Davis, The Wiener-Pitt phenomenon on semi-groups, Proc. Cambridge Philos. Soc. 59 (1963), 11-24.

3. E. Hewitt and K. Ross, Abstract harmonic analysis, Springer-Verlag, New York, 1963.

4. T. S. Liu, On vanishing algebras, Proc. Amer. Math. Soc. 14 (1963), 162-166.

5. - - On invariant subspaces, Quarterly J. Math. 14 (1963), 231-239.

6. R. Rigelhof, Norm decreasing homomorphisms of measure algebras, Trans. Amer. Math. Soc. 136 (1969), 361-371.

7. A. B. Simon, Vanishing algebras, Trans. Amer. Math. Soc. 92 (1959), 154-167.

McGill University, Montreal, Canada 